\title{
The properties of ultra-compact dwarf galaxies and their possible origin
}

\section{Michael Hilker*i}

Sternwarte der Universität Bonn, Germany

E-mail: mhilker@astro.uni-bonn.de

\section{Steffen Mieske}

Sternwarte der Universität Bonn, Germany

E-mail: smieskedastro.uni-bonn.de

\begin{abstract}
In this contribution the discovery and properties of ultra-compact dwarf galaxies are presented, and their possible origin is discussed. This new type of galaxy resides in the cores of galaxy clusters. In the fundamental plane diagram of stellar systems, the luminosity and kinematical and structural properties of ultra-compact dwarfs locate them inbetween globular clusters and small compact ellipticals (like M32). This regime would also be occupied by nuclei of dwarf ellipticals if they were isolated from their galactic envelope or by the merger product of super star-cluster complexes as they are found in strongly interacting galaxies. Future investigations of ultra-compact dwarfs in different environments have to show which of these formation scenarios is to be favoured.
\end{abstract}

Baryons in Dark Matter Halos

5-9 October 2004

Novigrad, Croatia

\footnotetext{
*Speaker.

${ }^{\dagger}$ Most of our work on UCDs is done in collaboration with: L. Infante, M.J. Drinkwater, M.D. Gregg, K. Bekki, W.J. Couch, H.C. Ferguson, J.B. Jones, A.M. Karick, S. Phillipps, \& M.J. West
} 


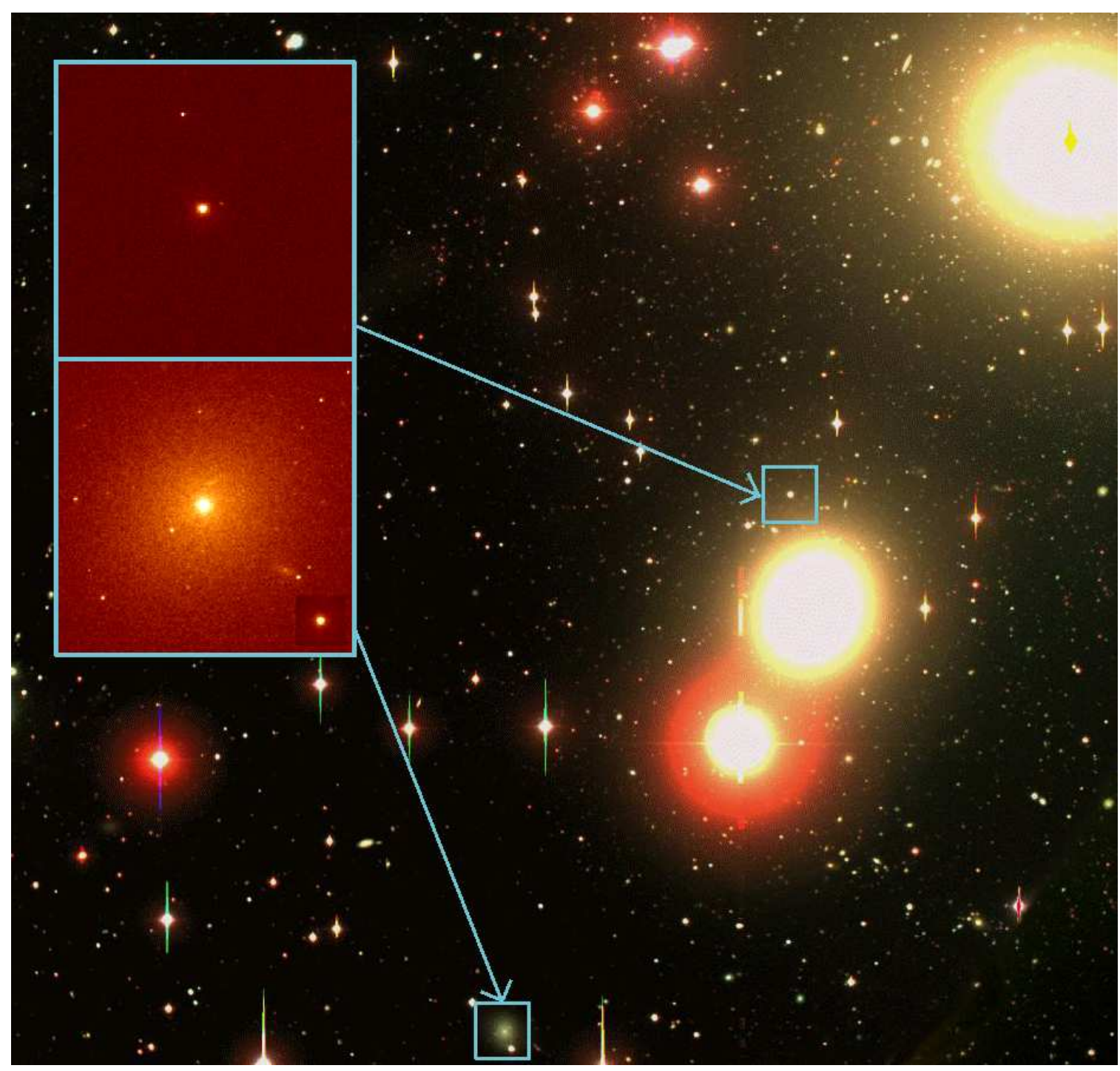

Figure 1: Central region of the Fornax cluster with the giant ellipticals NGC 1399 (upper right corner) and NGC 1404. The inlays show an ultra-compact dwarf galaxy (UCD) and a nucleated dwarf elliptical. This image is a 3-colour composite (BVI) and was taken with the $2.5 \mathrm{~m}$ DuPont telescope at Las Campanas Observatory in order to search for dwarf spheroidals in the cluster (Hilker et al. 2003).

\section{The discovery of ultra-compact dwarf galaxies}

Ultra-compact dwarf galaxies (UCDs) have recently been proposed as a new galaxy type (Drinkwater et al. 2003). They have first been discovered in the Fornax cluster (see Fig. 1).

In 1999, Hilker et al. presented the results of a spectroscopic survey of selected objects in the cluster core and reported on the confirmation of two new "very compact" members. They found them to "have photometric properties that can be explained by a very bright globular cluster as well as by a compact elliptical like M32". Also they suggested that these objects might "represent the nuclei of dissolved dwarf ellipticals", and stated that "it would be interesting to investigate, whether there are more objects of this kind hidden among the high surface brightness objects in the 

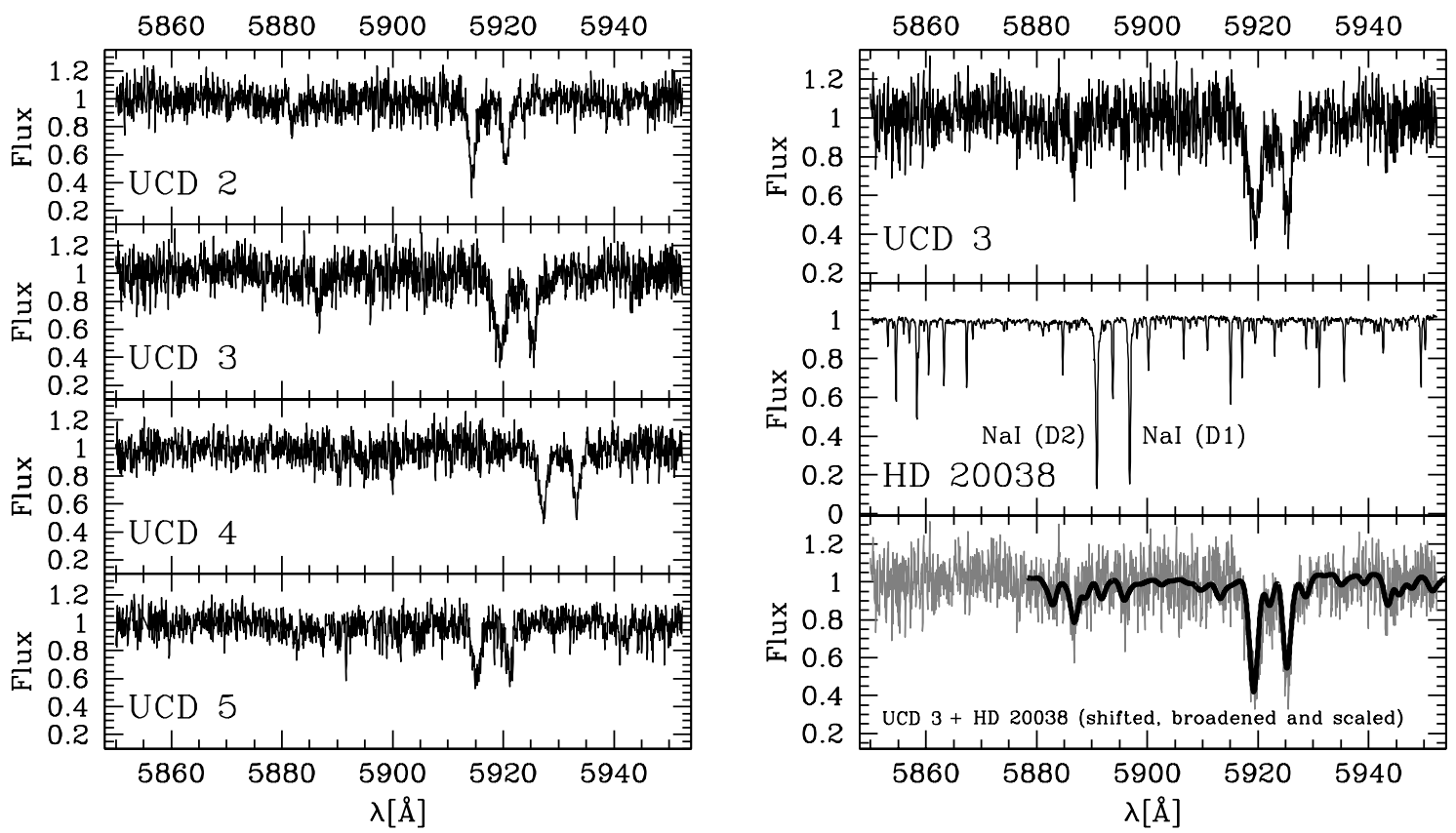

Figure 2: High resolution echelle spectra (UVES) that have been used for the determination of the internal velocity dispersion of the UCDs. On the left, the spectral region around the Na doublet is shown for four UCDs. On the right, the spectrum of UCD 3, a standard star, and the shifted, broadened and scaled spectrum of the standard star (grey curve) superimposed onto the UCD spectrum are shown.

central Fornax cluster". Indeed, one year later the results of a systematic spectroscopic survey in the central two degrees of the Fornax cluster (FCSS: Fornax Cluster Spectroscopic Survey, Drinkwater et al. 2000a) were published. Three further compact objects in the luminosity range $-12.2<M_{V}<$ -11.8 mag were discovered (Drinkwater et al. 2000b). This survey is unique in the sense that all objects in the two degree field, resolved as well as unresolved, have been targeted. Due to their compactness the five new Fornax members objects were named "ultra-compact dwarfs" (UCDs, Phillipps et al. 2001).

The absence of UCDs in the outer parts of the FCSS field suggests that these objects constitute a galaxy population that is preferentially found in the dense central region of galaxy clusters. No counterpart of a Fornax UCD has been found in the Local Group so far.

\section{Properties of ultra-compact dwarfs}

UCDs resemble globular clusters in their general appearance, but are up to 100 times more massive $\left(1-5 \times 10^{7} M_{\odot}\right)$ and slightly more extended $\left(r_{\text {eff }} \leq 30 \mathrm{pc}\right)$. Their luminosities are comparable to those of nuclei of dwarf ellipticals $\left(-13.5<M_{V}<-11.0\right)$, their photometric colours to those of metal-rich bulge globular clusters of giant ellipticals. From high resolution spectroscopy of four UCDs in the Fornax cluster, the internal velocity dispersion of their stars has been derived (see Fig. 2). Values found range from 20 to $40 \mathrm{~km} \mathrm{~s}^{-1}$. Using the sizes of the UCDs - derived from high resolution surface brightness profiles - their masses and mass-to-light ratios $\left(M / L_{B}\right)$ could be estimated. These are in the range of 2-4 in solar units (Drinkwater et al. 2003). This is slightly higher than the $M / L$ ratio of globular clusters, but much lower than found for dwarf spheroidal 


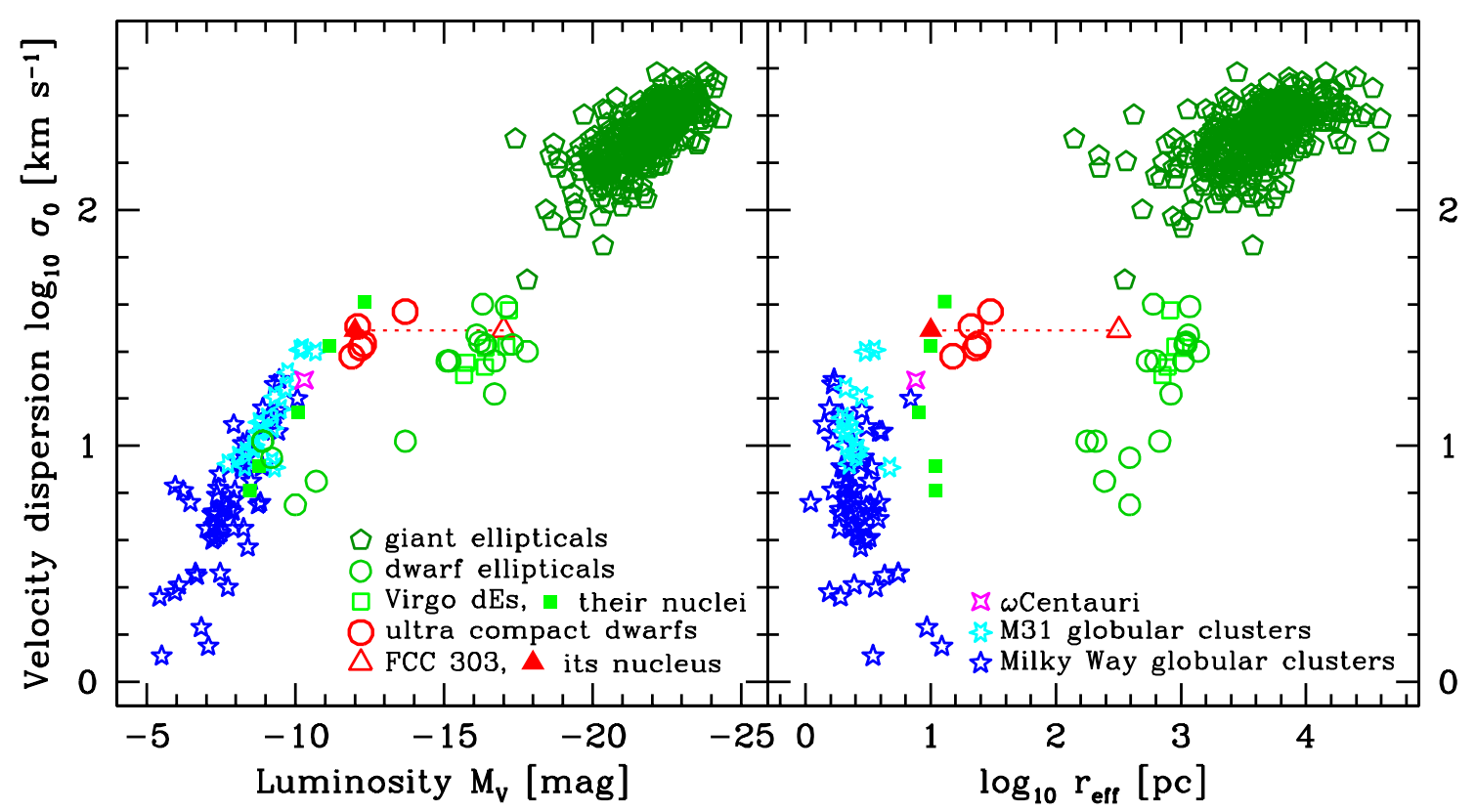

Figure 3: Fundamental plane, absolute magnitude $M_{V}$ vs. central velocity dispersion $\sigma_{0}$ (left) and effective radius $r_{\text {eff }}$ vs. $\sigma_{0}$ (right), for the indicated stellar systems. A scenario in which the stellar envelopes of nucleated dwarf galaxies are disrupted can explain the observed properties of the UCDs. See, for example, the location of FCC 303 and its nucleus (connected by a dashed line).

galaxies of similar mass. In the fundamental plane diagram of stellar systems (luminosity vs. velocity dispersion), the measured values of the UCDs are lying slightly off the relation for globular clusters, but are consistent with an extrapolation to fainter luminosities of the Faber-Jackson relation for elliptical galaxies (see Fig. 3). Other compact objects that are found at about the same location in the fundamental plane are nuclei of dwarf ellipticals (Geha et al. 2002), the nuclear clusters of late-type spirals (Walcher et al. 2004), as well as the young super star cluster W3 in NGC 7252, after evolving over a few Gyr (Maraston et al. 2003).

Bright UCDs $\left(M_{V}<-12.0\right)$ do not seem to exist in large numbers in galaxy clusters. The fainter ones can easily be confused with the bright globular clusters of the extraordinary rich globular cluster systems of the brightest cluster galaxies (i.e. Dirsch et al. 2003), and therefore their exact abundances are unclear. In order to investigate possible distinctions between UCDs and bright globular clusters, a systematic spectroscopic survey (Fornax Compact Object Survey: FCOS) of the brightest star clusters in the centre of the Fornax cluster was performed by Mieske et al. (2002, 2004). 54 new compact Fornax members were found. Bright compact objects $(V<20$ or $M_{V}<-11.4 \mathrm{mag}$ ), including the UCDs, have a higher mean radial velocity than faint compact objects $(V>20 \mathrm{mag})$ at $2 \sigma$ significance. The mean radial velocity of the bright compact objects is consistent with that of the dwarf galaxy population in Fornax, but inconsistent with that of the central galaxy NGC 1399's globular cluster systems. The compact objects show a trend of redder colour with increasing luminosity with a suggested slope similar to that of the well known colour-magnitude relation of dEs, but shifted about 0.2 mag redwards (see Fig. 4). The brightness distribution of compact objects observed in the FCOS shows a soft transition between UCDs and 


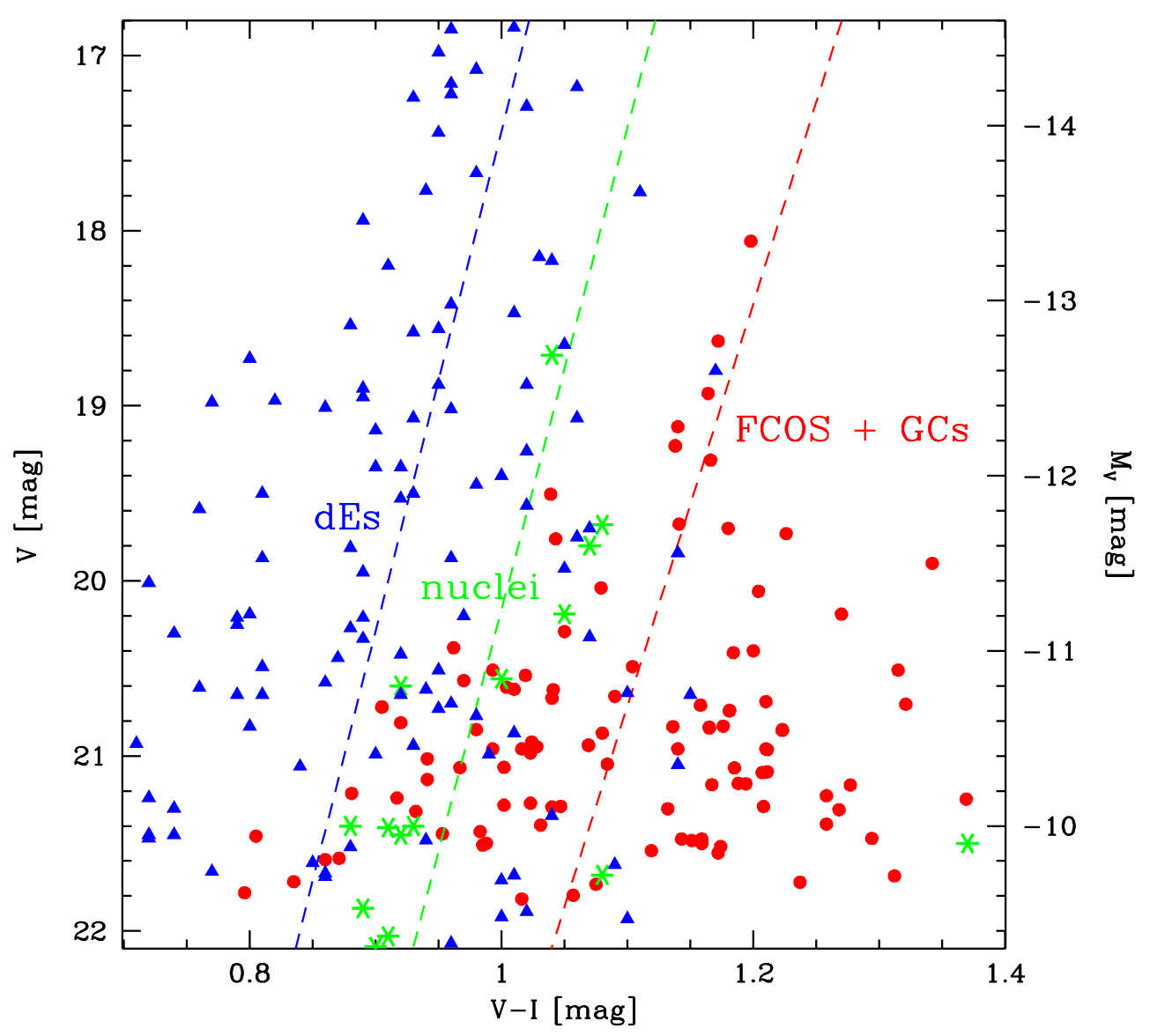

Figure 4: Colour magnitude diagram of dwarf ellipticals (blue triangles, from Hilker et al. 2003), nuclei of dEs (green asterisks, from Lotz et al. 2004), and compact objects (red dots, including bright globular clusters, from Mieske et al. 2004 and Dirsch et al. 2003). The lines are fits to the data points in the ranges $15.0<V_{\mathrm{dEs}}<22.0, V_{\text {nuclei }}<24.0$, and $V_{\text {compacts }}<21.0$.

GCs with a slight overpopulation with respect to the extrapolated very bright end of NGC 1399's GC luminosity function. The spatial distribution of bright compact objects within the cluster is, in comparison to the faint ones, more extended. Fainter than $V \simeq 20$ mag, the majority of the objects seem to be dominated by genuine GCs.

\section{Scenarios for the origin of UCDs}

Apart from the possibility that ultra-compact dwarfs are very extreme "ordinary" globular clusters, various formation scenarios have been brought forward to explain their origin and evolution see overview in Fig. 5). Two of them seem to be most promising: first, UCDs might be the remnant nuclei of dwarf galaxies that have been disrupted in the cluster environment (Bekki et al. 2003). Second, UCDs might have formed from the agglomeration of many young, massive star clusters that were created during an ancient merger event (Fellhauer \& Kroupa 2002). Numerical simulations have shown that the structural and kinematical properties of UCDs (i.e. their position in the fundamental plane) can be reproduced by both scenarios. 


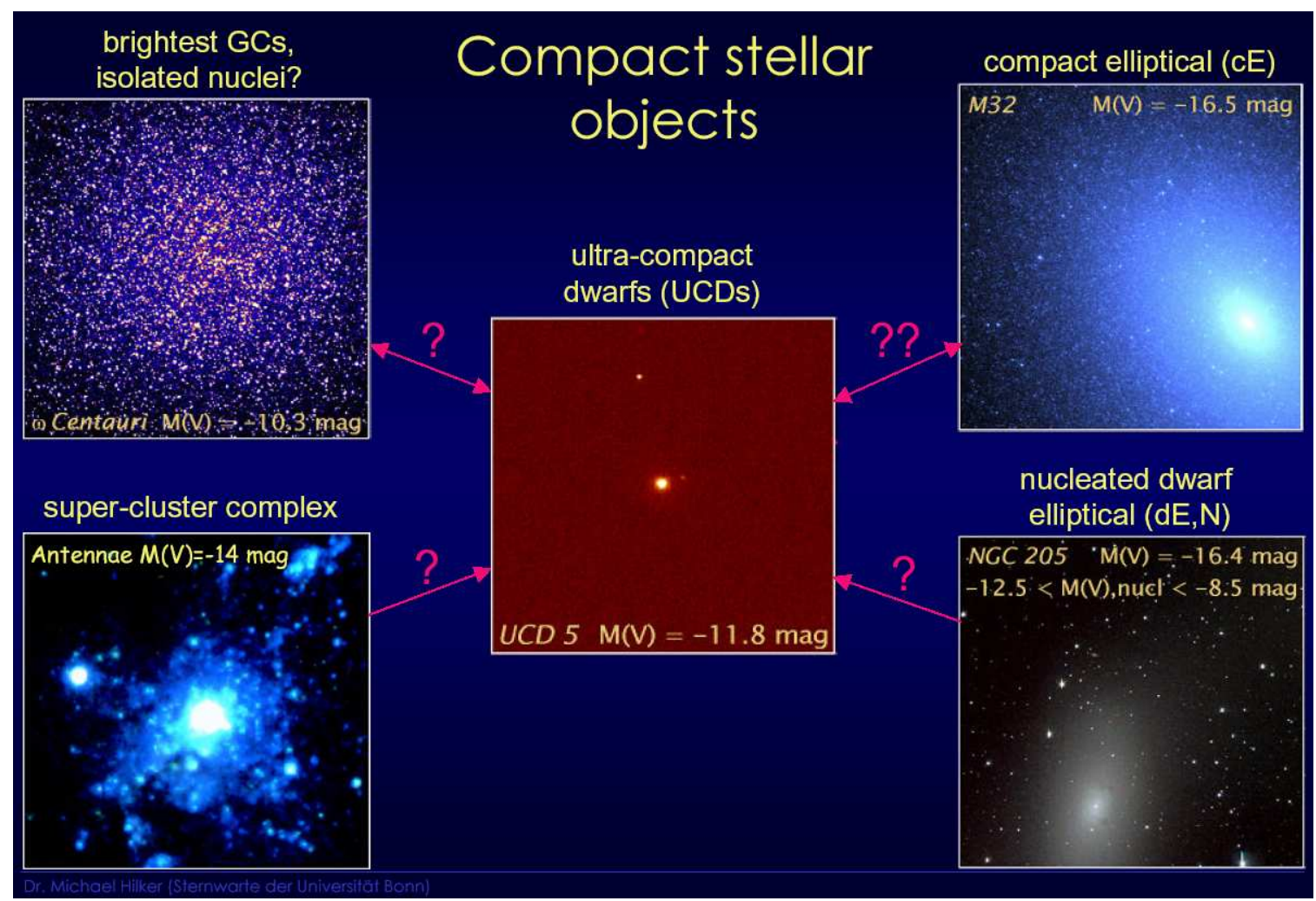

Figure 5: Compact stellar objects in comparison. How were the ultra-compact dwarfs formed? Are they genuine globular clusters, just more massive? Were they formed from merging super star clusters? Or the low mass end of compact ellipticals? Mostly favoured is a scenario in which they represent the isolated nuclei of threshed dwarf galaxies. Note that the objects shown are located at different distances.

The findings obtained from the FCOS data (see previous section) suggest that a substantial fraction of compact Fornax members brighter than $V \simeq 20$ mag might have been created by threshing dE,Ns (Bekki et al. 2003). The redder colour-magnitude relation of UCDs as compared to dEs would be expected from the stripping scenario if the stripped nuclei trace the colour-magnitude relation of their brighter progenitors. Also the number of UCDs in the Fornax cluster is roughly consistent with the predictions of Bekki et al. (2003) for stripped nuclei.

\section{Future research on UCDs}

In order to get further insight into the nature of UCDs more observations are needed. Three main questions should be addressed: 1) what are the ages and metallicities of their stellar populations? 2) what is the internal velocity dispersion of nuclei, UCDs, and bright globular clusters? Do they split up in the fundamental plane? and 3) what is the frequency of UCDs in different galactic environments? Do they exist in the field?

Concerning the first question, the spectroscopic measurement of line indices for dwarf nuclei and UCDs would solve existing age-metallicity degeneracies. HST imaging of dE,N-nuclei in Fornax/Virgo has shown that they are slightly bluer than UCDs (Lotz et al. 2004, and see Fig. 4). This suggests that UCDs either have higher metallicities and/or older integrated stellar populations 
than the present-day nuclei. The latter possibility is consistent with the threshing scenario if the ram-pressure stripping associated with the gravitational stripping of the UCD progenitor removes a large gas fraction from the nuclear region, thus inhibiting or lowering the efficiency of subsequent star formation events in the naked nucleus.

To answer the the second question, spatially resolved high resolution spectra for nuclei, UCDs, and GCs would be needed. Although challenging, one might also study the radial trend of their internal velocity dispersion. This should clarify whether UCDs are dark matter dominated (and thus of galaxian origin) or not.

For the third question, large spectroscopic surveys in a variety of galactic environments are needed. UCD candidates can be pre-selected by photometry. If tidal forces are the main triggers for the formation of UCDs, they should be extremely sparse in low density regions and most abundant in the densest and mostly evolved galaxy clusters.

\section{References}

[1] K. Bekki, W.J. Couch, M.J. Drinkwater, Y. Shioya, Galaxy threshing and the origin of ultra-compact dwarf galaxies in the Fornax cluster, MNRAS 344 (2003) 399 [ast ro-ph / 0308243 ].

[2] B. Dirsch, T. Richtler, D. Geisler, et al., The Globular Cluster System of NGC 1399. I. A Wide-Field Photometric Study, AJ 125 (2003) 1908 [astro-ph/ 0301223 ].

[3] M.J. Drinkwater, M.D. Gregg, M. Hilker, et al., A class of compact dwarf galaxies from disruptive processes in galaxy clusters, Nature 423 (2004) 519 [ast ro-ph/ 0306026 ].

[4] M.J. Drinkwater, J.B. Jones, M.D. Gregg, S. Phillipps, Compact stellar systems in the Fornax Cluster: Super-massive star clusters or extremely compact dwarf galaxies?, PASA 17 (2000b) 227 [astro-ph/0002003].

[5] M.J. Drinkwater, S. Phillipps, J.B. Jones, et al., The Fornax spectroscopic survey. I. Survey strategy and preliminary results on the redshift distribution of a complete sample of stars and galaxies, A\&A 355 (2000a) 900 [astro-ph/ 0001520 ].

[6] M. Fellhauer, P. Kroupa, The formation of ultracompact dwarf galaxies, MNRAS 330 (2002) 642 [astro-ph/0110621].

[7] M. Geha, P. Guhathakurta, R.P. van der Marel, Internal Dynamics, Structure, and Formation of Dwarf Elliptical Galaxies. I. A Keck/Hubble Space Telescope Study of Six Virgo Cluster Dwarf Galaxies, AJ 124 (2002) 3073 [astro-ph / 0206153 ].

[8] M. Hilker, L. Infante, G. Vieira, M. Kissler-Patig, T. Richtler, The central region of the Fornax cluster. II. Spectroscopy and radial velocities of member and background galaxies, A\&AS 134 (1999) 75 [astro-ph/9807144].

[9] M. Hilker, S. Mieske, L. Infante, Faint dwarf spheroidals in the Fornax Cluster. A flat luminosity function, A\&A 397 (2003) L9 [astro-ph/ 0212044 ].

[10] J.M. Lotz, B.W. Miller, H.C. Ferguson, The Colors of Dwarf Elliptical Galaxy Globular Cluster Systems, Nuclei, and Stellar Halos, ApJ 613 (2004) 262 [astro-ph / 04060 02].

[11] C. Maraston, N. Bastian, R.P. Saglia, et al., The dynamical mass of the young cluster W3 in NGC 7252: Heavy-Weight globular cluster or ultra compact dwarf galaxy?, A\&A 416 (2004) 467 [astro-ph/0311232]. 
[12] S. Mieske, M. Hilker, L. Infante, Ultra compact objects in the Fornax cluster of galaxies: Globular clusters or dwarf galaxies?, A\&A 383 (2002) 823 [astro-ph/ 0201011 ].

[13] S. Mieske, M. Hilker, L. Infante, Fornax compact object survey FCOS: On the nature of Ultra Compact Dwarf galaxies, A\&A 418 (2004) 445 [astro-ph/ 0401610 ].

[14] S. Phillipps, M.J. Drinkwater, M.D. Gregg, J.B. Jones, Ultracompact Dwarf Galaxies in the Fornax Cluster, ApJ 560 (2001) 201 [astro-ph/ 0106377 ].

[15] C.J. Walcher, R.P. van der Marel, D. McLaughlin, et al., Masses of star clusters in the nuclei of bulge-less spiral galaxies, ApJ (2004) [astro-ph/ 0409216 ]. 